\title{
A generalization of prime graphs of finite groups
}

\author{
Seiichi ABE and Nobuo IIYORI
}

(Received May 6, 1999)

\begin{abstract}
We investigate some properties of generalized prime graphs of finite groups, especially solvable graphs which is newly defined in this paper. A solvable graph of a finite simple group has a striking feature, that is, connected and incomplete. In the last section we give some applications of solvable graphs. We use the classification of finite simple groups in order to prove main theorems.
\end{abstract}

Key words: Sylow subgroup, prime graphs, simple groups.

\section{Introduction}

There are a lot of ways to characterize a finite group by orders of its elements. Considering a prime graph is one of such ways. In a prime graph $\Gamma(G)$ of a finite group $G$, edges $p$ and $q$ are defined to be joined when there exists an element $x$ of $G$ whose order is $p q$. This condition can be interpreted that $G$ includes a cyclic subgroup of order $p q$. So it seems natural to consider some other graphs in which the condition "being cyclic" is replaced to other ones. We will discuss solvable graphs which will be defined afterward of this paper and will show some applications of the graphs. Every group appearing in this paper is a finite group. Following the notation in Iiyori-Yamaki [4] and Williams [9], $\pi_{i}$ stands for the $i$ th connected components of prime graphs in tables of $[4,9]$ and we let $\operatorname{com}(G)$ stand for the number of connected components of prime graph of $G$.

\section{Definitions and Remarks}

Definition 1 Let $\Lambda$ be a set of positive rational integers. We denote $\Lambda$ graph by $\Gamma_{\Lambda}$ and the set of vertices of $\Gamma_{\Lambda}$ by $V_{\Lambda}$ which is the set of primes which divide an element of $\Lambda$. For vertices $p$ and $q$ of $\Gamma_{\Lambda}, p$ is joined to $q$ if and only if there exists an element $a$ in $\Lambda$ such that $p q \mid a$.

For example, let $\Lambda=\{6,7,30,33\}$. Then $\Gamma_{\Lambda}$ is the following;

1991 Mathematics Subject Classification : Primary 20D05, 20D06, 20 D20. 


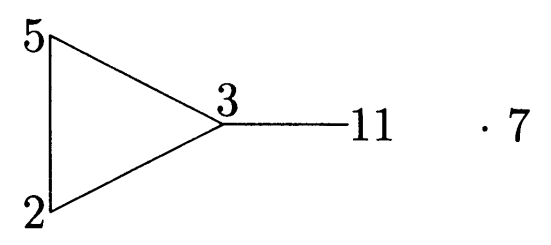

Definition 2 Let $\Xi$ be a group theoretical property. For a group $G, \mathcal{S}_{\Xi}(G)$ is the set of $\Xi$-subgroups of $G$. $\mathcal{S}_{\Xi}^{*}(G)$ is the set of $\Xi$-subgroups of $G$ which do not coincide with $G$. Let $\rho$ be a mapping of $\mathcal{S}_{\Xi}(G)$ to the set $\mathbb{N}$ of natural numbers.

$\Gamma_{\rho\left(\mathcal{S}_{\Xi}(G)\right)}$ stands for the $(\rho, \Xi)$-graph of $G$ and $\Gamma_{\rho\left(\mathcal{S}_{\Xi}^{*}(G)\right)}$ stands for the $(\rho, \Xi)^{*}$-graph of $G$.

We can consider several types of the mappings as follows: for $H \in$ $\mathcal{S}_{\Xi}(G)$, "ord" : $H \mapsto|H|$, "ind" $: H \mapsto|G: H|$, "conj" : $H \mapsto$ the number of conjugacy classes of $H$ to construct $H$ and so on.

Let $G$ be the alternating group $A_{5}$ of degree 5 and $\Xi$ be "solvable". Then an element of $\mathcal{S}_{\Xi}(G)$ is isomorphic to one of the following groups: the alternating $A_{4}$ of degree 4 , the dihedral group $D_{10}$ of order 10 , the symmetric group $S_{3}$ of degree 3 . Hence the (ord, $\Xi$ )-graph of $A_{5}$ is

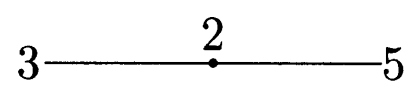

Let $\Xi^{\prime}$ be "abelian". Then the (ind, $\Xi^{\prime}$ )-graph of $A_{5}$ is as follows.

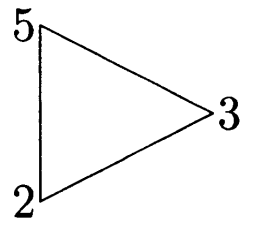

This time we focus on a mapping "ord" and disregard the rest. We denote the image of ord by $\operatorname{Ord} \Xi(G)$ for convenience.

$$
\operatorname{Ord} \Xi(G)=\operatorname{ord}\left(\mathcal{S}_{\Xi}(G)\right) \subseteq \mathbb{N}
$$

We simply call the (ord, $\Xi$ )-graph of $G$ the $\Xi$-graph of $G$. According to this rule, a prime graph $\Gamma(G)$ can be called a cyclic graph, which is denoted by $\Gamma_{c y c}(G)$. If $\Xi$ stands for "solvable", then we call the $\operatorname{Ord} \Xi(G)$-graph the solvable graph of a group $G$, which is denoted by $\Gamma_{\text {sol }}(G) . \Gamma_{n i l}(G)$, $\Gamma_{a b e l}(G)$ and so on can be defined in the same way where nil and abel stand for "nilpotent" and "abelian" respectively. It is easy to see that $\Gamma_{n i l}(G)$, 
$\Gamma_{\text {abel }}(G)$ and $\Gamma_{c y c}(G)$ are the same thing. Note that $\Gamma_{\text {sol }}(G)$ is different from $\Gamma_{c y c}(G)$ in general, although $V_{\text {sol }}(G)=V_{c y c}(G)$.

Example The solvable graph and the cyclic graph of $S_{6}(2)$ are drawn as below:
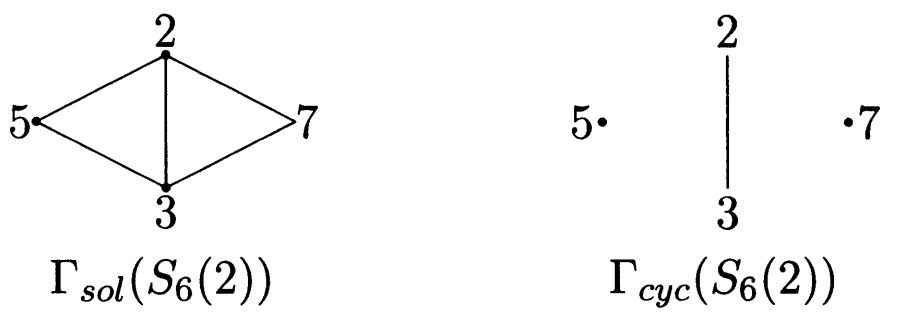

The following two lemmas are fundamental for our studies of solvable graphs of finite groups.

Lemma 1 Let $G$ be a group.

(1) If $G$ is not solvable, then $\Gamma_{\text {sol }}(G)=\Gamma_{\text {sol }}^{*}(G)$.

(2) If $G$ is solvable, then $\Gamma_{\text {sol }}(G)$ is complete.

(3) If $G$ is solvable and $|\pi(G)| \geq 3$, then $\Gamma_{\text {sol }}^{*}(G)$ is complete.

Lemma 2 Let $G$ be a group, $H$ a subgroup of $G$ and $N$ a normal subgroup of $G$.

(1) If $p$ and $q$ are not joined in $\Gamma_{\text {sol }}(G)$ for $p, q \in \pi(H)$, then $p$ and $q$ are not joined in $\Gamma_{\text {sol }}(H)$.

(2) Let $N$ be a normal subgroup of $G$. For $p \in \pi(N)$ and $q \in \pi(G)-\pi(N)$, $p$ and $q$ are joined in $\Gamma_{\text {sol }}(G)$.

(3) If $p$ and $q$ are not joined in $\Gamma_{\text {sol }}(G)$ for $p, q \in \pi(G / N)$, then $p$ and $q$ are not joined in $\Gamma_{\text {sol }}(G / N)$.

Proof. It is easy to see that (1) holds. By the Frattini argument, $N_{G}(Q) N=G$ for a Sylow $q$-subgroup of $N$. For a Sylow $p$-subgroup $P$ of $N_{G}(Q), P Q$ is a solvable subgroup of $G$ such that $p q|| P Q \mid$. This implies (2). Suppose that $p$ and $q$ are joined in $\Gamma_{s o l}(G / N)$. Then there exists a solvable subgroup $H / N$ of $G / N$ such that $\pi(H / N)=\{p, q\}$. If $|N|$ and $p q$ are coprime, then there exists a subgroup $K$ of $G$ such that $H=K N$, $K \cap N=1, K \simeq H / N$ by Schur-Zassenhaus' theorem. Since $K$ is a solvable group, $p$ and $q$ are forced to be joined in $\Gamma_{s o l}(G)$. This is a contradiction. The proof is complete. 


\section{Some Results on Solvable Graphs}

Theorem 1 Let $G$ be a non abelian simple group. Then $\Gamma_{\text {sol }}(G)$ is connected.

We divide the proof into following three cases:

(1) $G$ is isomorphic to the alternating group of degree $n(n \geq 5)$,

(2) $G$ is a simple group of Lie type,

(3) $G$ is a sporadic finite simple group.

We need some lemmas to prove Theorem 1.

Lemma 3 (Williams [9]) Let $G$ be a non abelian simple group such that $\operatorname{com}(G) \geq 2$. Then the following hold.

(1) $G$ has a Hall $\pi_{i}$-subgroup $H_{i}$ for a connected component $\pi_{i}(i \geq 2)$ of the prime graph of $G$

(2) $H_{i}$ is an isolated abelian subgroup of $G$.

Lemma 4 Let $G$ be a non abelian simple group such that $\operatorname{com}(G) \geq 2$ and $H_{i}$ is an isolated $\pi_{i}$-subgroup. Then $H_{i}$ is a proper subgroup of $N_{G}\left(H_{i}\right)$ for $i \geq 2$.

Proof. Suppose $N_{G}\left(H_{i}\right)=H_{i}$. Since $H_{i}$ is a Hall $\pi_{i}$-subgroup of $G$, a Sylow $p$-subgroup $P$ of $H_{i}$ is that of $G$ for any $p \in \pi(G)$. Since $P$ is the unique Sylow $p$-subgroup of $H_{i}$ by Lemma $2, N_{G}\left(H_{i}\right) \subseteq N_{G}(P)$. If $N_{G}\left(H_{i}\right)$ is a proper subgroup of $N_{G}(P)$, then this contradicts Lemma 2. Hence $N_{G}\left(H_{i}\right)=N_{G}(P)$. Since $H_{i}$ is an abelian subgroup, then $H_{i}=Z\left(N_{G}(P)\right)$. By Burnside's transfer theorem, there exists a normal complement of $P$. This contradicts the simplicity of $G$.

Corollary 1 Let $G$ be a non abelian simple group such that $\operatorname{com}(G) \leq 2$. Then $\Gamma_{\text {sol }}(G)$ is connected.

Proof. As we mentioned in the definition of $\Gamma_{\text {sol }}(G)$, if two primes which divide the order of $G$ are joined in the prime graph of $G$, they are also joined in $\Gamma_{\text {sol }}(G)$. Therefore we may assume that $\operatorname{com}(G)=2$. For $p \in \pi_{2}$, there exists $q \in \pi\left(N_{G}\left(H_{2}\right)\right)-\pi_{2}$ by Lemma 2. Since $\pi(G)$ is decomposed to $\pi_{1}$ and $\pi_{2}, q \in \pi_{1}$. Hence $\Gamma_{\text {sol }}(G)$ is connected.

Proof of Theorem 1 .

Case: $G \simeq A_{n}(n \geq 5)$

For $n=5,6$ see Atlas [2]. For $n \geq 7, \operatorname{com}(G) \leq 2$ by Williams [9]. The 
The number $i$ in Tables of this paper corresponds to the $i$ th prime graph component $\pi_{i}$ in Williams [9].

Table 1. Three components

\begin{tabular}{c|l|c|c|l}
\hline group & $i$ & $\left|H_{i}\right|$ & $\left|N_{G}\left(H_{i}\right): H_{i}\right|$ & \\
\hline$A_{1}(q)$ & 2 & $q$ & $(q-1) / 2$ & $q \equiv 1(4)$ \\
& 3 & $(p+1) / 2$ & 2 & \\
\hline${ }^{2} D_{p}\left(3^{2}\right)$ & 2 & $\left(3^{p-1}+1\right) / 2$ & $a$ & $(a, 2(p-1)) \neq 1$ \\
& 3 & $\left(3^{p}+1\right) / 4$ & $b$ & $(b, 2 p) \neq 1$ \\
\hline$E_{7}(2)$ & 2 & 73 & $2 \cdot 3^{2}$ & \\
& 3 & 127 & $s$ & $2 \cdot 7 \mid s$ \\
\hline$E_{7}(3)$ & 2 & 757 & $2 \cdot 3^{2}$ & \\
& 3 & 1093 & $t$ & $2 \mid t$ \\
\hline$F_{4}(q)$ & 2 & $q^{4}+1$ & $2^{3}$ & $2 \mid q$ \\
& 3 & $q^{4}-q^{2}+1$ & $2^{2} \cdot 3$ & \\
\hline${ }^{2} F_{4}(q)$ & 2 & $q^{2}+q m+q+m+1$ & $2^{3}$ & $q=2^{2 k+1}(k \geq 1)$, \\
& & & & $m=2^{k+1}$ \\
& 3 & $q^{2}-q m+q+m+1$ & $2^{2} \cdot 3$ & \\
\hline$G_{2}(q)$ & 2 & $q^{2}+e q-1$ & $2 \cdot 3$ & $q \equiv e(3), e= \pm 1$ \\
& 3 & $q^{2}+e q+1 / 3$ & $2 \cdot 3$ & \\
\hline$G_{2}(q)$ & 2 & $q^{2}+q-1$ & $2 \cdot 3$ & $q \equiv 0(3)$ \\
& 3 & $q^{2}-q+1$ & $2 \cdot 3$ & \\
\hline${ }^{2} G_{2}(q)$ & 2 & $q+3 m+1$ & $2 \cdot 3$ & $q=3^{2 k+1}(k \geq 1)$, \\
& & & & $m=3^{k}$ \\
& 3 & $q-3 m+1$ & $2 \cdot 3$ & \\
\hline
\end{tabular}

theorem holds for $G$ by Corollary 1 .

Case: $G$ is a simple group of Lie type If $\operatorname{com}(G) \leq 2$, then the theorem holds for $G$ by Corollary 1. For every group $G$ such that $\operatorname{com}(G) \geq 3$ except ${ }^{2} D_{p}\left(3^{2}\right)$ and $A_{2}(4)$, Tables 1,2 show that 2 divides $\left|N_{G}\left(H_{i}\right): H_{i}\right|$ for any isolated subgroup $H_{i}$ corresponding to a prime graph component $\pi_{i}$ of $G$. This implies that the theorem holds for $G$. See Atlas [2] for $G \simeq A_{2}(4)$. Let $\simeq{ }^{2} D_{p}\left(3^{2}\right)$. Both $a$ and $b$ in the Table 1 can be divided by elements of $\pi_{1}$. 
Table 2. Four and Five components

\begin{tabular}{c|c|c|c|l}
\hline group & $i$ & $\left|H_{i}\right|$ & $\left|N_{G}\left(H_{i}\right): H_{i}\right|$ & \\
\hline$A_{2}(4)$ & 2 & $3^{2}$ & 2 & \\
& 3 & 5 & 2 & \\
& 4 & 7 & 3 & \\
\hline${ }^{2} B_{2}(q)$ & 2 & $q-1$ & 2 & $q=2^{2 k+1}(k \geq 1)$, \\
& & & & $m=2^{k+1}$ \\
& 3 & $q+m+1$ & $2^{2}$ & \\
& 4 & $q-m+1$ & $2^{2}$ & \\
\hline${ }^{2} B_{2}(q)$ & 2 & $q-1$ & 2 & $q=2^{2 k+1}(k \geq 1)$, \\
& & & & $m=2^{k+1}$ \\
& 3 & $q+m+1$ & $2^{2}$ & \\
& 4 & $q-m+1$ & $2^{2}$ & \\
\hline$E_{8}(q)$ & 2 & $q^{8}+q^{7}-q^{5}-q^{4}+q^{3}+q+1$ & 30 & $q \equiv 2,3(5)$ \\
& 3 & $q^{8}-q^{7}+q^{5}-q^{4}+q^{3}-q+1$ & $2 \cdot 3 \cdot 5$ & \\
& 4 & $q^{8}-q^{4}+1$ & $2^{2} \cdot 3$ & \\
\hline$E_{8}(q)$ & 2 & $q^{8}+q^{7}-q^{5}-q^{4}+q^{3}+q+1$ & 30 & $q \equiv 0,1,4(5)$ \\
& 3 & $q^{8}-q^{7}+q^{5}-q^{4}+q^{3}-q+1$ & $2 \cdot 3 \cdot 5$ & \\
& 4 & $q^{8}-q^{6}+q^{4}-q^{2}+1$ & $2 \cdot 3 \cdot 5$ & \\
& 5 & $q^{8}-q^{4}+1$ & $2^{2} \cdot 3$ & \\
\hline
\end{tabular}

Case: $G$ is a sporadic simple group

In table IIc in Williams [9], Monster $M$ has 4 prime graph components, say $\pi_{2}=\{41\}, \pi_{3}=\{59\}$ and $\pi_{4}=\{71\}$. Since prime divisors of orders of automorphisms groups of a Sylow 41-subgroup, a Sylow 59-subgroup and a Sylow 71-subgroup of $M$ belong to the prime graph component $\pi_{1}, \Gamma_{\text {sol }}(M)$ is connected.

In table IIc in Williams [9], Mathieu group $M_{24}$ of degree 24 has three prime graph components, say $\pi_{2}=\{11\}$ and $\pi_{3}=\{23\}$. Since the order of the normalizer of a Sylow 23-subgroup of $M_{24}$ is $11 \cdot 23, \Gamma_{\text {sol }}\left(M_{24}\right)$ is connected. The connectivity of $\Gamma_{\text {sol }}(G)$ can be shown in the same way for other sporadic simple groups.

This completes the proof of Theorem 1 .

Corollary 2 Let $G$ be a finite group. Then $\Gamma_{\text {sol }}(G)$ is connected. 
Theorem 2 Let $G$ be a non-abelian simple group. Then $\Gamma_{\text {sol }}(G)$ is an incomplete graph.

By the classification of finite simple groups, $G$ is isomorphic to one of the followings:

(1) alternating groups $A_{n}(n \geq 5)$,

(2) classical simple groups, i.e., $\operatorname{PSL}_{n}(q), \operatorname{PSU}_{n}(q), \operatorname{PSp}_{n}(q), \mathrm{P}_{2 n}^{ \pm}(q)$, $\mathrm{P} \Omega_{2 n+1}(q)$,

(3) other simple groups of Lie type, or

(4) 26 sporadic finite simple groups.

We will prove our theorem for each case.

Case: $G=$ an alternating group $A_{n}(n \geq 5)$

Lemma 5 Let $n \neq 6,10$. Then there exist at least two primes between $n / 2$ and $n$.

Proof. By Brandl-Shi [1], there exist more than 6 primes between $n / 2$ and $n$ for $n \geq 47$. Therefore the lemma is straightforward.

Suppose that $\Gamma_{\text {sol }}\left(A_{n}\right)$ is a complete graph. By Lemma 5, there exists primes $p, q$ such that $n / 2<p, q \leq n$. Since $\Gamma_{\text {sol }}\left(A_{n}\right)$ is a complete graph, there exists a subgroup $H$ of order $p q$ of $A_{n}$. Let $x, y \in H$ such that $o(x)=p, o(y)=q$. Then $\langle x\rangle$ or $\langle y\rangle$ is a normal subgroup of $H$. Note that Aut $(\langle x\rangle) \simeq \mathbb{Z}_{p-1}$ and $\operatorname{Aut}(\langle y\rangle) \simeq \mathbb{Z}_{q-1} . q \backslash p-1$ and $p \nmid q-1$ lead that $H$ is an abelian subgroup of $A_{n}$ by Burnside's transfer theorem. Since the order of the centralizer of $x$ is coprime to $q$, we have a contradiction. Hence $\Gamma_{\text {sol }}\left(A_{n}\right)$ is not a complete graph.

Case: $G=$ a classical simple group.

First we will prove the incompleteness of $\Gamma_{\text {sol }}(\mathrm{GL}(n, q))(n \geq 4)$. In this case we denote $\operatorname{GL}(n, q)(n \geq 4)$ by $L_{n}$. Let $\Phi_{m}(x)$ be the cyclotomic polynomial of index $m$.

Lemma 6 Let $m>n / 2$.

(1) Let $(q, m) \neq(2,6)$. There exist a cyclic subgroup $C_{m}$ of order $q^{m}-1$ and a Hall subgroup $H_{m}$ of order $h_{m}(q)=\Phi_{m}(q) /\left(\Phi_{m}(q), m\right)$ of $L_{n}$ such that $H_{m} \subset C_{m}$.

(2) Let $x \in H_{m}$. Then there exists a subgroup $T$ of $L_{n}$ such that $C_{L_{n}}(x) \subseteq$ $C_{m} T$ and $T \simeq L_{n-m}$.

(3) $\quad N_{L_{n}}\left(H_{m}\right) \simeq\left(C_{m}: m\right) \times T$. 
Proof. Since $\mathbb{F}_{q^{m}}$ can be regarded as a vector space over $\mathbb{F}_{q}$ of dimension $m, \mathbb{F}_{q^{m}}^{\times} \subset \mathrm{GL}\left(\mathbb{F}_{q^{m}}\right)$. Since $h_{m}(q)$ is a Hall divisor of $L_{n},(1)$ holds. (2) is shown in the same manner of liyori [5]. It is easy to see that $N_{\mathrm{GL}\left(\mathbb{F}_{q^{m}}\right)}\left(\mathbb{F}_{q^{m}}^{\times}\right) \simeq \mathbb{F}_{q^{m}}^{\times}: m$. Then we have (3). The proof is complete.

Suppose that $r$ and $s$ are joined in $\Gamma_{\text {sol }}\left(L_{n}\right)$ for $r \in \pi\left(H_{n}\right)$ and $s \in \pi\left(H_{l}\right)$ $(n>l>n / 2)$. Then there exists a subgroup of $L_{n}$ such that $\pi(K)=$ $\{r, s\}$. Lemma 6 (1) yields that $R$ and $S$ are cyclic for $R \in \operatorname{Syl}_{r}(K)$ and $S \in \operatorname{Syl}_{s}(K)$. At least one of the following happens; (i) $R \neq N_{K}(R)$, (ii) $S \neq N_{K}(S)$, or (iii) $R=N_{K}(R)$ and $S=N_{K}(S)$. By Burnside's transfer theorem, $R=N_{K}(R)$ implies that $S$ is a normal subgroup of $K$, which contradicts $S=N_{K}(S)$. Therefore the case (iii) never happen.

Suppose that (ii) holds. Since $N_{K}(S) \subset N_{L_{n}}(S) \simeq C_{l}: l \times L_{n-l}$ and $\left(\left|C_{n}\right| \cdot\left|L_{n-l}\right|, r\right)=1, r$ divides $l . r \leq l$ and $q^{r-1}-1 \equiv 0(\bmod r)$ yield $r \mid\left(h_{n}(q), q^{r-1}-1\right)$, which contradicts that $h_{n}(q)$ is a Hall divisor of $L_{n}$. If (i) holds, then $\pi\left(N_{K}(R)\right)=\{r, s\}$. We may assume that $R$ is a subgroup of $H_{n}$. Lemma 6 leads $N_{L_{n}}(R) \simeq C_{n}: n . N_{K}(R) \subset N_{L_{n}}(R) \simeq C_{n}: n$ and $\left(\left|C_{n}\right|, s\right)=1$ imply $s \mid n$. We note that there exists $a \in \mathbb{Z}$ such that $n=a s$ and $q^{s-1}-1 \equiv 0(\bmod r)$. If $a \geq 2$, then $l<n / 2$. This contradicts the choice of $l$. Thus we have $s=n$ and $l=n-1$, which lead the following lemma.

Lemma 7 (1) $h_{n}(q)=1$ if and only if $(q, n)=(2,6)$.

(2) Let $n \geq 4$. Suppose that $h_{n}(q), h_{l}(q), h_{n-1}(q) \neq 1$. Let $r \in \pi\left(H_{n}\right)$ and $s \in \pi\left(H_{l}\right)(n / 2<l<n-1)$. Then $r$ and $s$ are not joined in $\Gamma_{\text {sol }}\left(L_{n}\right)$. $r \in \pi\left(H_{n}\right)$ and $s \in \pi\left(H_{n-1}\right)$ are joined in $\Gamma_{\text {sol }}\left(L_{n}\right)$ if and only if $s=n$.

(3) Let $q=p^{u}$ for a prime $p$. Suppose that $r \in \pi\left(H_{n}\right)$ and $p \nmid n$. Then $r$ and $p$ are not joined in $\Gamma_{\text {sol }}\left(L_{n}\right)$.

Proof. Let $L_{n}=\mathrm{GL}(n, q)$ act on a vector space $V$ of dimension $n$ naturally. Suppose that there exists a subgroup $U$ of $L_{n}$ such that $\pi(U)=\{p, r\}$. Since $N_{L_{n}}(R) \simeq\left(q^{n}-1\right): n$ and $\left(\left(q^{n}-1\right) n, p\right)=1, N_{U}(R)=R$ for $R \in \operatorname{Syl}_{r}(U)$. By Burnside's transfer theorem, a Sylow $p$-subgroup $P$ of $U$ is a normal subgroup of $U$. Put $W=\left\{v \in V \mid v^{x}=v\right.$ for all $\left.x \in P\right\}$. Note $W \neq 0$. For $y \in U$ and $w \in W,\left(w^{y}\right)^{x}=\left(w^{y x y^{-1}}\right)^{y}=w^{y}$ implies that $\left.W^{y} \subseteq W .(r, \mid \operatorname{GL}(n-1, q)) \mid\right)=1$ leads $W=V$. This contradicts $P \neq 1$. We have (3) 
Now we are going to prove Theorem 2 for classical simple groups.

[1] $G=\operatorname{PSL}_{n}(q)$.

Lemma 7 implies that the theorem holds for $G=\operatorname{PSL}_{n}(q)$ for $n \geq 4$ unless $(q, n)=(2,6)$.

For $G=\mathrm{PSL}_{2}(q)$, see Suzuki [8].

Suppose $G=\operatorname{PSL}_{3}(q)$. If $p \neq 3$, then $p$ and a prime which divides $h_{3}(q)$ are not joined in $\Gamma_{\text {sol }}\left(\mathrm{PSL}_{3}(q)\right)$ by Lemma 7. If $p=3$, then namely $q$ is a power of $3, G=\mathrm{SL}_{3}(q)$ includes an isolated cyclic subgroup $H$ of order $q^{2}+$ $q+1$ by Williams [9]. Suppose that $r$ and 2 are joined in $\Gamma_{s o l}(G)$ for a prime $r$ such that $r|| H \mid$. Then there exists a subgroup $J$ of $G$ such that $\pi(J)=$ $\{2, r\} . N_{G}(H) \simeq H: 3$ and $(|H: 3|, 2)=1$ imply that a Sylow 2-subgroup $Q$ is a normal subgroup of $J$ by Burnside's transfer theorem. There exists a Sylow 2-subgroup $B$ of $\mathrm{GL}_{3}(q)$ such that $Q \subseteq B$. Let $V$ be a natural $\mathrm{GL}_{3}(q)$-module of dimension 3 . Since there exists a canonical embedding of $\mathrm{GL}_{1}(q) \times \mathrm{GL}_{2}(q)$ to $\mathrm{GL}_{3}(q)$ and $\left|\mathrm{GL}_{1}(q)\right|_{2} \cdot\left|\mathrm{GL}_{2}(q)\right|_{2}=\left|\mathrm{GL}_{3}(q)\right|_{2}$, then $V=W_{1} \oplus W_{2}$ as $B$-module such that $\operatorname{dim} W_{1}=1$. Since $W_{1}$ is a $Q$ module which is generated by an element $w$ of $W_{1}$, there exists an eigen value $\lambda_{y} \in \mathbb{F}_{q}$ such that $w^{y}=\lambda_{y} w$ for $y \in Q$. For $z \in R, w^{z y z^{-1}}=\lambda_{z y z^{-1}} w$ leads $\left(w^{z}\right)^{y}=\lambda_{z y z^{-1}} w^{z}$. Thus $\left\langle w^{z}\right\rangle$ is a $Q$-module. The $R$-irreduciblity of $V$ implies $V=W_{1} \oplus W_{1}^{z} \oplus W_{1}^{z^{2}}$. Since $V$ is a faithful $Q$-module and there exists an embedding of $Q$ to $\mathrm{GL}_{1}(q)^{3}, \Omega_{1}(Z(Q))$ is isomorphic to a subgroup of $\mathbb{Z}_{2}^{3}$. $Q \subset \mathrm{SL}_{3}(q)$ leads $|Q| \mid 4$. Since $\Omega_{1}(Z(Q)) R$ is a Frobenius group, $r$ divides $\left|\Omega_{1}(Z(Q))\right|-1$. This implies that $r=3$, which is contradiction. Thus $r$ and 2 are not joined in $\Gamma_{\text {sol }}(G)$.

Let $G=\mathrm{PSL}_{6}(2)$. Suppose that 7 and 31 are joined in $\Gamma_{\text {sol }}(G)$. There exists a subgroup $A$ of $G$ such $\pi(A)=\{31,7\} .|G|_{31,7}=31 \cdot 7^{2}$ leads that $A$ is an abelian group. This contradicts that $G$ has an isolated subgroup of order 31 by Iiyori-Yamaki [3]. Thus 7 and 31 are not joined in $\Gamma_{\text {sol }}(G)$. Hence the theorem holds for $G=\operatorname{PSL}_{n}(q)$ except $(n, q)=(2,2),(2,3)$.

[2] $G=\operatorname{PSp}_{2 n}(q)$.

For $n \geq 3$ except $(q, n)=(2,3),(2,4)$, we have that $h_{2 n}(q) \neq 1$, $h_{2 n-2}(q) \neq 1$ and that $h_{2 n}(q) h_{2 n-2}(q)|| \operatorname{Sp}_{2 n}(q) \mid$. For primes $r$ and $s$ such that $r \mid h_{2 n}(q)$ and $s \mid h_{2 n-2}(q), r$ and $s$ are not joined in $\Gamma_{s o l}\left(\mathrm{GL}_{n}(q)\right)$ by Lemma 7. Which implies that they are not joined in $\Gamma_{\text {sol }}\left(\operatorname{PSp}_{n}(q)\right)$ by Lemma 2. If $(q, n)=(2,3)$ i.e. $G=\operatorname{PSp}_{6}(2)$, then 5 and 7 are not joined by Atlas [2]. Let $(q, n)=(2,4)$ i.e. $G=\mathrm{PSp}_{8}(2)$. Suppose that there exists 
a solvable subgroup $E$ of order $7 \cdot 17$. Then $E$ is an abelian group. This contradicts that $G$ contains no element of order $7 \cdot 17$ (see Atlas [2]).

Let $G=\operatorname{PSp}_{4}(q)$ and $V$ be a natural module over $\mathbb{F}_{q}$ of dimemsion 4. For a prime $r$ such that $r \mid h_{4}(q)=q^{2}+1, r$ and $p$ are not joined in $\Gamma_{\text {sol }}\left(\mathrm{GL}_{4}(q)\right)$ by Lemma 7 if $p \neq 2$. If $p=2$, then $G$ includes an isolated cyclic subgroup $B$ of order $q^{2}+1$. We will show that $a \in \pi(B)$ is not joined to $r$ which divides $q+1$. Suppose that $r$ and $a$ are joined in $\Gamma_{s o l}\left(\operatorname{Sp}_{4}(q)\right)$. Then there exists a subgroup $T$ of $\operatorname{PSp}_{4}(q)$ such that $\pi(T)=\{a, r\}$. We may assume that a Sylow a-subgroup $R$ of $T$ is a subgroup of $B \cdot N_{\operatorname{Sp}_{4}(q)}(B) \simeq$ $\left(q^{2}+1\right): 2$ and $\left(\left|\left(q^{2}+1\right): 2\right|, r\right)=1$ lead that $R$ is a normal subgroup of $T$. Let $G_{0}=\mathrm{GL}_{4}(q) .\left|G_{0}\right|_{q+1}=(q+1)^{2}$ yields that $R$ is conjugate to a subgroup of

$$
\left\{\left(\begin{array}{cc}
Z_{1} & \\
& Z_{2}
\end{array}\right)\left|Z_{1}, Z_{2} \in \mathrm{GL}_{2}(q), o\left(Z_{1}\right), o\left(Z_{2}\right)\right| q+1\right\} .
$$

Hence $V=W_{1} \oplus W_{2}$ as $R$-module. Note that $W_{i}(i=1,2)$ is an irreducilbe $R$-module of dimension 2. If $R$ is not cyclic, then $N_{G_{0}}(R)$ is isomorphic to a subgroup of $\mathrm{GL}_{2}(q) \backslash S_{2} .\left(a,\left|\mathrm{GL}_{2}(q)<S_{2}\right|\right)=1$ leads that $R$ is not a normal subgroup of $T$, which is a contradiction. Therefore $R$ is a cyclic group. $C_{G}(R)$ includes a subgroup $L$ which is isomorphic to $\mathbb{Z}_{q+1} \times \mathbb{Z}_{q+1}$. Since $B$ is isolated, $a \backslash\left|C_{G}(R)\right| .\left[T, C_{G}(R)\right] \subset C_{G}(R)$ yields that $K=T C_{G}(R)$ is a subgroup of $G$. By the Frattini argument, $K=N_{K}\left(R_{0}\right) C_{G}(R)$ for $R_{0} \in \operatorname{Syl}_{r}\left(C_{G}(R)\right) . \quad R_{0} \simeq \mathbb{Z}_{|q+1|_{r}} \times \mathbb{Z}_{|q+1|_{r}}$ leads that $N_{K}(R)$ includes a subgroup $T_{0} \simeq \mathbb{Z}_{r}^{2}: a$. This attributes to the case in which $R$ is not cyclic. Hence $r$ and $a$ are not joined in $\Gamma_{\text {sol }}\left(\operatorname{Sp}_{4}(q)\right)$.

$$
\text { [3] } G=\mathrm{P} \Omega_{2 n}^{+}(q)(n \geq 4) \text {. }
$$

Lemma 8 Let $G=\operatorname{GL}_{n}(q)(n \geq 10)$. For primes $r$ and $s$ such that $r \mid h_{n-2}(q)$ and $s \mid h_{n-4}(q), r$ and $s$ are not joined in $G$.

Proof. Suppose that $r$ and $s$ are joined in $\Gamma_{\text {sol }}(G)$.Then there exists a subgroup $H$ such that $\pi(H)=\{r, s\}$. Lemma 7 implies $\left|N_{G}(R)\right|=\left(q^{n-2}-\right.$ 1) $(n-2)\left|\mathrm{GL}_{2}(q)\right|$ and $\left|N_{G}(S)\right|=\left(q^{n-4}-1\right)(n-4)\left|\mathrm{GL}_{4}(q)\right|$ for $R \in \operatorname{Syl}_{r}(H)$ and $S \in \operatorname{Syl}_{s}(H)$. Since there exists no element of order $r s, r \mid(n-4)$ or $s \mid(n-2)$ by Burnside's transfer theorem. If $s \mid(n-2)$, then Fermat's theorem leads $s=(n-2) . s \mid\left(q^{n-3}-1\right)$ and $\left(q^{n-3}-1, h_{n-4}(q)\right)=1$ yield a contradiction. Then $r \mid(n-4)$. Fermat's theorem leads $r=n-4$ and $r \mid\left(q^{n-5}-1\right)$. This is a contradiction. Therefore $r$ and $s$ are not joined in 
$\Gamma_{\text {sol }}(G)$. This completes the proof.

By the previous lemma, $\Gamma_{\text {sol }}\left(\mathrm{P}_{2 n}^{+}(q)\right)$ is not a complete graph for $n \geq 5$. Suppose that $n=4$. For $G=\mathrm{P} \Omega_{8}^{+}(2), \Gamma_{\text {sol }}(G)$ is not complete as drawn below:

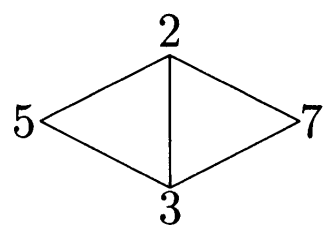

Let $G_{0}=\mathrm{GL}_{8}(q)(q \neq 2), G=\mathrm{P} \Omega_{8}^{+}(q)$ and $V$ a natural $G_{0}$-module of dimension 8. Suppose that $r$ and $s$ are joined in $\Gamma_{s o l}(G)$ for primes $r$ and $s$ such that $r \mid h_{6}(q)$ and $s \mid h_{4}(q)$. Then there exists a subgroup $H$ such that $\pi(H)=\{r, s\}$. For $R \in \operatorname{Syl}_{r}(H)$ and $S \in \operatorname{Syl}_{s}(H),\left|N_{G_{0}}(R)\right|=$ $\left(q^{6}-1\right) 6\left|\mathrm{GL}_{2}(q)\right|$ by Lemma 7. $s \backslash 76$ implies that $S$ is the normal $r$ complement of $R$ by Burnside's transfer theorem. Since a Sylow $s$-subgroup of $G_{0}$ is conjugate to a subgroup of

$$
\left\{\left(\begin{array}{cc}
Z_{1} & \\
& Z_{2}
\end{array}\right)\left|Z_{1}, Z_{2} \in \mathrm{GL}_{4}(q), o\left(Z_{1}\right), o\left(Z_{2}\right)\right| h_{4}(q)\right\},
$$

there exist $S$-modules $W_{i}(i=1,2)$ such that $V=W_{1} \oplus W_{2}$ and $\operatorname{dim} W_{i}=$ $4(i=1,2)$. Note that at least one of $W_{i}(i=1,2)$ is $S$-irreducible. We may assume that $W_{1}$ is irreducible. Suppose that there exists $w, v \in W_{1}$ such that $w^{y} \neq w$ and $v^{y}=v$ for $y \in R$. For $z \in S, w^{y z y^{-1}} \in W_{1}$ implies that $W_{1}^{y}$ is an $S$-module. $o(y) \mid h_{6}(q)$ yields $W_{1}^{y} \neq W_{1}$. The irreducibility of $W_{1}$ contradicts that $W_{1}^{y} \cap W_{1}$ is an $S$-module and $W_{1}^{y} \cap W_{1} \neq 0$. Hence $w^{y} \neq w$ for $w \in W_{1}-\{0\}$. Since $W_{1}$ is not $R$-invariant, the $S$-irreducibility of $W_{1}$ implies $V=\left\langle W_{1}^{y} \mid y \in R\right\rangle$. Then $W_{2}$ is $S$-irreducible by KrullRemak-Schmidt's theorem. $V$ decomposes into an irreducible $R$-module of dimension 6 and two trivial modules. Then there exist $w, v \in W_{2}$ such that $w^{y} \neq w$ and $v^{y}=v . W_{2}^{y} \cap W_{2}$ is an $S$-module and $W_{2} \neq W_{2}^{y} \cap W_{2} \neq 0$. This contradicts the irreducibility of $W_{2}$. Hence $r$ and $s$ are not joined in $\Gamma_{\text {sol }}(G)$.

$$
\text { [4] } G=\mathrm{P} \Omega_{2 n}^{-}(q)(n \geq 4) \text {. }
$$

$O_{2 n}^{-}(q)$ is a subgroup of $\mathrm{GL}_{2 n}(q)$ and $h_{2 n}(q)|| G \mid$. Lemma 7 yields the incompleteness of $G$ unless $(q, n) \neq(2,4)$. Therefore $\Gamma_{\text {sol }}\left(\mathrm{P} \Omega_{8}^{-}(2)\right)$ is not complete by Atlas [2]. 
[5] $G=\mathrm{P} \Omega_{2 n+1}(q)(n \geq 3)$.

By Lemma 7 and $8, \Gamma_{\text {sol }}\left(\mathrm{P} \Omega_{2 n+1}(q)\right)$ is not complete if $n \geq 4$. We may assume $G=\mathrm{P} \Omega_{7}(q)$ and $q$ is odd. Let $G_{1}=O_{7}(q)$, which is a subgroup of $O_{8}^{+}(q)$. Note that $h_{6}(q) \neq 1$ and $h_{4}(q) \neq 1$. For primes $r$ and $s$ such that $r \mid h_{6}(q)$ and $s \mid h_{4}(q), r$ and $s$ are not joined in $\Gamma_{s o l}\left(\mathrm{P} \Omega_{8}^{+}(q)\right) . h_{6}(q) h_{4}(q)|| G_{1} \mid$ implies that $r$ and $s$ are not joined in $\Gamma_{\text {sol }}\left(\mathrm{P} \Omega_{7}(q)\right)$ by Lemma 2. Thus the theorem holds for $\left.\mathrm{P} \Omega_{2 n+1}(q)\right)$ for $n \geq 3$.

[6] $G=\operatorname{PSU}_{n}(q)(n \geq 3$ and $(q, n) \neq(2,3))$

Let $G_{0}=\mathrm{GL}_{n}\left(q^{2}\right)$ and $G_{1}=\mathrm{U}_{n}(q)$. Then $G_{1}$ is a subgroup of $G_{0}$. Suppose that $n$ is odd. $h_{n}\left(q^{2}\right)|| G \mid$ and Lemma 7 yield that the incompleteness of $\Gamma_{\text {sol }}(G)$ if $p \nmid n$. Suppose $p \mid n$. If $n \geq 5$, Lemma 7 yields that $r$ and $s$ are not joined in $\Gamma_{\text {sol }}(G)$ for primes $r$ and $s$ such that $r \mid h_{n}(q)$ and $s \mid h_{n-2}(q)$. Hence we may assume that $G=\operatorname{PSU}_{3}\left(3^{t}\right)((q, n) \neq(2,3))$ for a positive integer $t$. Since $r$ and 2 are not joined in $\Gamma_{\text {sol }}\left(\mathrm{PSL}_{3}\left(3^{2 t}\right)\right)$, the theorem holds for $n=$ odd. Suppose that $n$ is even. If $n \geq 6$, then Lemma 8 implies the incompleteness of $\Gamma_{\text {sol }}(G)$.

Let $G=\operatorname{PSU}_{4}(q), G_{0}=\mathrm{GL}_{4}\left(q^{2}\right)$ and $V$ a vector space over $\mathbb{F}_{q^{2}}$ of dimension 4 .

Suppose that $r$ and $s$ are joined in $\Gamma_{s o l}(G)$ for primes $r$ and $s$ such that $r \mid h_{3}\left(q^{2}\right)$ and $s \mid\left(q^{2}+1\right) /\left(q^{2}+1,2\right)$. Then there exists a subgroup $H$ of $G$ such that $\pi(H)=\{r, s\}$. Let $R$ be a Sylow $r$-subgroup of $G$ and $S$ a Sylow $s$-subgroup of $G$. There exists a subgroup $H_{0}$ of $G_{0}$ such that $H_{0} \simeq \mathrm{GL}_{2}\left(q^{2}\right) \times \mathrm{GL}_{2}\left(q^{2}\right)$ and $\operatorname{Syl}_{r}\left(H_{0}\right) \subseteq \operatorname{Syl}_{r}\left(G_{0}\right)$. Since $H$ is conjugate to

$$
\left\{\left(\begin{array}{cc}
Z_{1} & \\
& Z_{2}
\end{array}\right)\left|Z_{1}, Z_{2} \in \mathrm{GL}_{2}\left(q^{2}\right), o\left(Z_{1}\right), o\left(Z_{2}\right)\right|\left(q^{2}+1\right) /\left(q^{2}+1,2\right)\right\},
$$

there exist $S$-modules $W_{i}(i=1,2)$ such that $V=W_{1} \oplus W_{2}, \operatorname{dim} W_{i}=2$ $(i=1,2)$ and that at least one of $W_{i}(i=1,2)$ is $S$-irreducible. By the same observations for $R$, there exist $S$-modules $W_{i}(i=3,4)$ such that $V=W_{3} \oplus W_{4}, \operatorname{dim} W_{3}=3, \operatorname{dim} W_{4}=1$ and that $W_{i}(i=3,4)$ is $R$ irreducible. The same argument for $\Gamma_{\text {sol }}\left(\mathrm{P} \Omega_{8}^{+}(q)\right)$ gives a contradiction. The theorem holds for $G=\operatorname{PSU}_{n}(q)(n \geq 3$ and $(q, n) \neq(2,3))$.

The theorem is proved for every classical simple group.

\section{Case: $G=$ other simple groups of Lie type.}

Let $G$ be a simple group of Lie type which is not a classical simple group. Suppose $G$ is not ${ }^{3} D_{4}(q)$ nor ${ }^{2} F_{4}(2)^{\prime}$. 
Table 3. Hall abelian subgroups

\begin{tabular}{|c|c|c|c|}
\hline group & $|H|$ & $\left|N_{G}(H): H\right|$ & \\
\hline${ }^{2} B_{2}(q)$ & $\begin{array}{c}q-1 \\
q+m+1 \\
q-m+1\end{array}$ & $\begin{array}{l}2 \\
2^{2} \\
2^{2}\end{array}$ & $\begin{array}{l}q=2^{2 k+1}(k \geq 1) \\
m=2^{k+1}\end{array}$ \\
\hline${ }^{3} D_{4}(q)$ & $q^{4}-q^{2}+1$ & $2^{2}$ & \\
\hline$E_{6}(q)$ & $\begin{array}{c}\left(q^{6}+q^{3}+1\right) /(3, q-1) \\
\left(q^{4}+q^{3}+q^{2}+q+1\right) /(5, q-1) \\
q^{4}-q^{2}+1 \\
\left(q^{4}+1\right) /(2, q-1)\end{array}$ & $\begin{array}{c}3^{2} \\
2 \cdot 5 \\
2^{2} \cdot 3 \\
2^{2} \cdot 3\end{array}$ & \\
\hline${ }^{2} E_{6}(q)$ & $\begin{array}{c}\left(q^{6}-q^{3}+1\right) /(3, q+1) \\
\left(q^{4}-q^{3}+q^{2}-q+1\right) /(5, q+1) \\
q^{4}-q^{2}+1 \\
\left(q^{4}+1\right) /(2, q-1)\end{array}$ & $\begin{array}{c}3^{2} \\
2 \cdot 5 \\
2^{2} \cdot 3 \\
2^{2} \cdot 3\end{array}$ & \\
\hline$E_{7}(q)$ & $\begin{array}{l}\left(q^{6}+q^{3}+1\right)(q+1) \\
\left(q^{6}-q^{3}+1\right)(q-1)\end{array}$ & $\begin{array}{l}2 \cdot 3^{2} \\
2 \cdot 3^{2}\end{array}$ & \\
\hline$E_{8}(q)$ & $\begin{array}{c}\left(q^{8}+q^{7}-q^{5}-q^{4}-q^{3}+q+1\right) \\
\left(q^{8}-q^{7}+q^{5}-q^{4}+q^{3}-q+1\right) \\
q^{8}-q^{4}+1\end{array}$ & $\begin{array}{l}2 \cdot 3 \cdot 5 \\
2 \cdot 3 \cdot 5 \\
2^{2} \cdot 3\end{array}$ & $q \equiv 2,3(5)$ \\
\hline$E_{8}(q)$ & $\begin{array}{c}\left(q^{8}+q^{7}+q^{5}+q^{3}-q+1\right) \\
\left(q^{8}+q^{7}-q^{5}-q^{4}-q^{3}+q+1\right) \\
\left(q^{8}-q^{7}+q^{5}-q^{4}+q^{3}-q+1\right) \\
q^{8}-q^{4}+1 \\
\end{array}$ & $\begin{array}{c}2 \cdot 3 \cdot 5 \\
2 \cdot 3 \cdot 5 \\
2 \cdot 3 \cdot 5 \\
2^{2} \cdot 3 \\
\end{array}$ & $q \equiv 0,1,4(5)$ \\
\hline$F_{4}(q)$ & $\begin{array}{c}q^{4}+1 \\
q^{4}-q^{2}+1\end{array}$ & $\begin{array}{c}2^{3} \\
2^{2} \cdot 3\end{array}$ & $2 \mid q$ \\
\hline${ }^{2} F_{4}(q)$ & $\begin{array}{l}q^{2}+q m+q+m+1 \\
q^{2}-q m+q+m+1\end{array}$ & $\begin{array}{c}2^{3} \\
2^{2} \cdot 3\end{array}$ & $\begin{array}{l}q=2^{2 k+1}(k \geq 1) \\
m=2^{k+1}\end{array}$ \\
\hline${ }^{2} F_{4}^{\prime}(2)$ & 13 & $2 \cdot 3$ & \\
\hline$G_{2}(q)$ & $\begin{array}{c}q^{2}+e q-1 \\
q^{2}+e q+1 / 3 \\
\end{array}$ & $\begin{array}{l}2 \cdot 3 \\
2 \cdot 3\end{array}$ & $q \equiv e(3), e= \pm 1$ \\
\hline$G_{2}(q)$ & $\begin{array}{l}q^{2}+q-1 \\
q^{2}-q+1\end{array}$ & $\begin{array}{l}2 \cdot 3 \\
2 \cdot 3\end{array}$ & $q \equiv 0(3)$ \\
\hline${ }^{2} G_{2}(q)$ & $\begin{array}{l}q+3 m+1 \\
q-3 m+1\end{array}$ & $\begin{array}{l}2 \cdot 3 \\
2 \cdot 3\end{array}$ & $\begin{array}{l}q=3^{2 k+1}(k \geq 1) \\
m=3^{k}\end{array}$ \\
\hline
\end{tabular}


$G$ includes two cyclic subgroups $H_{i}(i=1,2)$ by Table $3 . H_{i}$ has the following properties:

(P1) $H_{i}$ includes a non trivial Hall cyclic subgroup $H_{i}^{0}$ of $G$, such that $\pi\left(H_{1}\right) \cap \pi\left(H_{2}^{0}\right)=\emptyset$ and $\pi\left(H_{2}\right) \cap \pi\left(H_{1}^{0}\right)=\emptyset$,

(P2) $C_{G}(x)=H_{i}$ for $x \in\left(H_{i}^{0}\right)^{\sharp}$,

(P3) $\quad\left(\left|N_{G}\left(H_{1}^{0}\right): H_{1}\right|,\left|H_{2}\right|\right)=\left(\left|N_{G}\left(H_{2}^{0}\right): H_{2}\right|,\left|H_{1}\right|\right)=1$

(P4) $H_{i}^{0}$ is a T.I. set of $G$

Suppose that $r$ and $s$ are joined in $\Gamma_{\text {sol }}(G)$ for $r \in \pi\left(H_{1}^{0}\right)$ and $s \in$ $\pi\left(H_{2}^{0}\right)$. Then there exists a subgroup $M$ of $G$ such that $\pi\left(H_{1}\right)=\{r, s\}$. For $R \in \operatorname{Syl}_{r}(M)$ and $S \in \operatorname{Syl}_{s}(M)$, Sylow's theorem allows $H_{1}$ to include $R$. Suppose $R \neq N_{M}(R)$. Then there exists an $s$-element $x$ in $N_{M}(R) . R^{x}=R$ and (P4) imply $x \in N_{G}\left(H_{1}^{0}\right)$, which contradicts (P3). $R=N_{M}(R)$ yields that $S$ is a normal subgroup of $M$ by Burnside's transfer theorem. The same argument shows that $R$ is a normal subgroup of $M$. Then $M$ is an abelian subgroup. This contradicts (P1) and (P2). Therefore $r$ and $s$ are not joined in $\Gamma_{\text {sol }}(G)$.

$\Gamma_{\text {sol }}\left({ }^{2} F_{4}(2)^{\prime}\right)$ is not complete as drawn below by Atlas [2]:

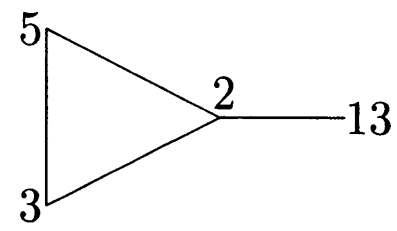

Let $G={ }^{3} D_{4}(q)$. Let $q=p^{a}$. By Kleidman [6], a subgroup $H$ of $G$ such that $\left(h_{12 a}(p),|H|\right) \neq 1$ is isomorphic to a subgroup of $\left(q^{4}-q^{2}+1\right): 4$. This implies that a prime divisor of $h_{12 a}(p)$ is joined only to 2 . Therefore the theorem holds for $G$.

Case: $G=$ one of 26 sporadic simple groups.

Atlas [2] yields the theorem for $G$.

The proof of the theorem is completed.

Theorem 3 Let $G$ be a finite group and $p, q \in \pi(G) . \quad p$ and $q$ are not joined in $\Gamma_{\text {sol }}(G)$ if and only if there exists a series of normal subgroups of G

$$
G \unrhd N \unrhd M \unrhd 1,
$$

such that $G / N$ and $M$ are $\{p, q\}^{\prime}$-group and $N / M$ is a non abelian simple group such that $p$ and $q$ are not joined in $\Gamma_{\text {sol }}(N / M)$. 
Proof. We will prove our theorem by induction on the order of $G$. Suppose that $p$ and $q$ are not joined in $\Gamma_{\text {sol }}(G)$. If $G$ is a non ableian simple group, then our theorem obviously holds. Let $M_{1}$ be a minimal normal subgroup of $G$. By Lemma 2, one of the following two cases happens; (1) $p, q \notin \pi\left(M_{1}\right)$, (2) $p, q \in \pi\left(M_{1}\right)$ and $p, q \notin \pi\left(G / M_{1}\right)$.

Case (1): By Lemma 2, $p$ and $q$ are not joined in $\Gamma_{\text {sol }}\left(G / M_{1}\right)$. By the assumption of induction, our theorem holds for $G / M_{1}$. Then there exists a series of normal subgroups of $G / M_{1}$

$$
G / M_{1} \unrhd \tilde{N} / M_{1} \unrhd \tilde{M} / M_{1} \unrhd 1,
$$

such that $\left(G / M_{1}\right) /\left(\tilde{N} / M_{1}\right)$ and $\tilde{M} / M_{1}$ are $\{p, q\}^{\prime}$-group and $\left(\tilde{N} / M_{1}\right) /$ $\left(\tilde{M} / M_{1}\right)$ is a non abelian simple group such that $p$ and $q$ are not joined in $\Gamma_{\text {sol }}\left(\tilde{N} / M_{1}\right) /\left(\tilde{M} / M_{1}\right)$. This implies the theorem holds for $G$.

Case (2): If $M_{1}$ is isomorphic to the direct product of $n$ copies of a cyclic group of order $r$ for a prime $r$. Since $r$ is the only prime which divides $\left|M_{1}\right|$. This contradicts $p, q \in \pi\left(M_{1}\right)$. Therefore $M_{1}$ is isomorphic to the direct product of $n$ copies of a non abelian simple group $S$. Suppose $n \geq 2$. Then $\Gamma_{\text {sol }}(G)$ should be a complete graph. Hence $n=1$ and our theorem holds for $G$.

We will show the converse. Suppose that $p$ and $q$ are joined in $\Gamma_{\text {sol }}(G)$. Then there exists a subgroup $H$ of $G$ such that $\pi(H)=\{p, q\}$. Since $H M / M \simeq H /(H \cap M)$ is a subgroup of $G / M$ such that $\pi(H M / M)=\{p, q\}$. Any $p$ - and $q$-elements of $G / M$ are contained in $N / M$ since $p, q \notin \pi(N / M)$. Therefore $p$ and $q$ are joined in $\Gamma_{s o l}(N / M)$. This is a contradiction. The proof is complete.

\section{Applications}

Let $X_{n}=\{i \in \mathbb{N} \mid 1 \leq i \leq n\}$. We say that $X_{n}$ is consecutive up to $n$. The following theorems are shown as applications of prime graphs.

Theorem 4 (Brandl-Shi) [1] Let $G$ be a finite group. If $\operatorname{Ord}_{c y c}(G)$ is consecutive up to $n$, Then $n \leq 8$ and $G$ can be classified.

Using the argument in Brandl-Shi [1], the similar result for $\operatorname{Ord}_{a b e l}(G)$ was shown by N. Chigira. 
Theorem 5 Let $G$ be a finite group. If $\operatorname{Ord}_{\text {sol }}(G)$ is consecutive up to $n$, Then $G \simeq \mathbb{Z}_{2}$ or 1 .

Proof. Suppose $n \geq 3$. Then there exists a prime $p$ such that $n / 2<$ $p \leq n$. Since $\Gamma_{\text {sol }}(G)$ is connected for a finite group by Theorem $1, p$ is joined to another prime $q \in X_{n}$. But $n<p q \in X_{n}$, which contradicts the maximumness of $n$ in $X_{n}$. Therefore $n=1,2$. The proof of our theorem is complete.

Lemma 9 Let $\operatorname{Ord}_{\text {sol }}^{*}(G)$ be consecutive up to $n$. Then $n \leq 4$.

Proof. Suppose $n>4$. Then there exists a prime $p$ such that $n / 2<p \leq n$ which is isolated in $\Gamma_{\text {sol }}^{*}(G)$, namely $p$ is not joined to no other vertices. So $\Gamma_{\text {sol }}^{*}(G)$ is disconnected. By Lemma 1 $(3),|\pi(G)| \leq 2$ holds. Thus $\pi(G) \subseteq$ $\{2,3\}$ and $n \leq 4$.

Theorem 6 If $\operatorname{Ord}_{\text {sol }}^{*}(G)$ is consecutive up to $n$, Then $n \leq 4$ and

$$
\begin{array}{rlrl}
G & \simeq A_{4} & & (n=4) \\
& \simeq S_{3}, \mathbb{Z}_{6} & & (n=3) \\
& \simeq \mathbb{Z}_{2} \times \mathbb{Z}_{2}, \mathbb{Z}_{4} & (n=2) \\
& \simeq 1, \mathbb{Z}_{p} & (n=1)
\end{array}
$$

for any prime $p$.

Proof. By lemma 9, $\pi(G)=\{2,3\}$ and $|G|=12$ if $n=4$. By Lemma 10, $G \simeq A_{4}$. Since 6 is not contained in $\operatorname{Ord}_{\text {sol }}^{*}(G), 2$ and 3 are not joined in $\Gamma_{\text {cyc }}(G)$. This implies $\operatorname{com}(G) \geq 2$. $G$ is a Frobenius group or 2-Frobenius group by Kegel-Gruenberg [8]. So $G \simeq A_{4}$. It is easy to check the rest of cases.

\section{References}

[1] Brandl R. and Shi W., Finite groups whose element orders are consecutive integer. J. Algebra 143 (1991), 388-400.

[2] Conway J.H. et al, Atlas of Finite Groups. Oxford Univ. Press (Cleandon), London/New York 1985.

[3] Iiyori N. and Yamaki H., A conjecture of Frobenius and the simple groups of Lie Type III. J. Algebra 145 (1992), 329-332.

[4] Iiyori N. and Yamaki H., Prime graph components of the simple group of Lie type over the field of even characteristic. J. Algebra 155 (1993), 335-343. 
[5] Iiyori N., A conjecture of Frobenius and the simple groups of Lie Type IV. J. Algebra 154 (1993), 188-214.

[6] Kleidman P.B., The maximal subgroups of the Steinberg triality groups ${ }^{3} D_{4}(q)$ and their automorphism groups. J. Algebra 115 (1988), 182-199.

[7] Suzuki M., On a class of doubly transitive groups. Ann. of Math. 75 (1962), 105145.

[ 8] Suzuki M., Group Theory I, II. Springer, Berlin-Heidelberg-New York, 1982.

[9] Williams J.S., Prime graph components of finite groups. J. Algebra 69 (1981), 487-513.

[10] Yamaki H., A conjecture of Frobenius and the simple groups of Lie Type I. Arch. Math. 42 (1984), 344-347; II, J. Algebra 96 (1985), 391-396.

Seiichi Abe

Department of Mathematics

Faculty of Education

Yamaguchi University

Yamaguchi 753-8512, Japan

E-mail: c2125@sty.sv.cc.yamaguchi-u.ac.jp

Nobuo Iiyori

Department of Mathematics

Faculty of Education

Yamaguchi University

Yamaguchi 753-8512, Japan

E-mail: iiyori@po.cc.yamaguchi-u.ac.jp 